This is the final peer-reviewed accepted manuscript of:

A 3D-centered information system for the documentation of a complex restoration intervention / Fabrizio Ivan Apollonio, Vilma Basilissi, Marco Callieri, Matteo Dellepiane, Marco Gaiani, Federico Ponchio, Francesca Rizzo, Angelo Raffaele Rubino, Roberto Scopigno, Giorgio Sobra', Journal of Cultural Heritage, Volume 29, 2018, Pages 89-99.

The final published version is available online at: http://dx.doi.org/10.1016/i.culher.2017.07.010

Rights / License:

The terms and conditions for the reuse of this version of the manuscript are specified in the publishing policy. For all terms of use and more information see the publisher's website.

This item was downloaded from IRIS Università di Bologna (https://cris.unibo.it/)

When citing, please refer to the published version. 


\title{
A 3D-centered information system for the documentation of a complex restoration intervention
}

\author{
Fabrizio Ivan Apollonio a, Vilma Basilissi b ${ }^{\text {, }}$ Marco Callieri ${ }^{c}$, Matteo Dellepiane ${ }^{\mathrm{c}, *}$, Marco \\ Gaiani a ${ }^{a}$, Federico Ponchio ${ }^{c}$, Francesca Rizzo ${ }^{\mathrm{a}}$, Angelo Raffaele Rubino ${ }^{\mathrm{b}}$, Roberto \\ Scopigno ${ }^{\text {, }}$, Giorgio Sobra' b \\ a Department of Architecture, University of Bologna, 40136 Bologna, Italy \\ ${ }^{\mathrm{b}}$ Istituto Superiore per la Conservazione ed il Restauro, MIBACT, 00153 Roma, Italy \\ ' Istituto di Scienza e Tecnologie dell'Informazione "Alessandro Faedo" (ISTI), Consiglio Nazionale delle Ricerche (CNR), via Moruzzi 1, 56124 Pisa, Italy
}

Restoration is becoming a quite complex process: a large number of internal and external variables co-exist and may impair it. Among these, the large number of professionals involved and the huge amount of documentation produced can heavily affect the quality of the intervention as well as the possibility to have systemic and informed interventions in the future. In particular, a standardized method for storing restoration data and accessing them is still lacking, and the use of new technologies is still limited and/or not scalable. The paper describes the process of designing and testing an information system (IS) based on three-dimensional (3D) data, aimed to support the restoration of Neptune's Fountain in Bologna. In preparation of the restoration, a major effort was carried out to design and implement a web-based IS able to host all of the data produced, to allow the conservation-restoration specialists to interact on-site with an accurate 3D representation of the elements of the fountain, and to directly reference all information and data produced on the geometry of the model. The paper focuses on the challenges and adopted solutions related to the use of 3D models and the data mapping on 3D surfaces in the context of restoration documentation. Highly detailed visualizations of the models, easy navigation, and usable functionalities to add information directly on the 3D model have been achieved by extending the available solutions and by implementing new mechanisms to overcome the limitations of WebGL and remote rendering. Neptune IS' development has been extensively experimented in a real context of use. Results and knowledge from the experimentation currently represents the basis for evolving Neptune IS into a possible generic and flexible platform for documentation management in the field of restoration and related methodologies.

\section{Research aim}

Documentation organization and accessibility are important tasks in all restoration processes. This work aims to explore a new methodology for the organization, management and access of all the data produced during complex restoration processes supported by a 3D-based information system (IS). The system exploits the 3D model of the object under restoration as the pivotal element and the central reference space for the whole work. The paper then discusses the results coming from a real context experimentation of the IS in a restoration project and describes the future development

\footnotetext{
* Corresponding author.

E-mail address: matteo.dellepiane@isti.cnr.it (M. Dellepiane).
}

of the system based on the results coming from the experimentation.

Currently, the IS permits storing data in real time, by mapping them over a high-fidelity, digital 3D model of the artwork and inserting them in the system database. The IS relies on web standards and employs web technologies (HTML5 and WebGL) so that it can be used from everywhere and by everyone via the Internet. The system supports not only the online insertion of information, but also its consultation and preservation.

\section{Introduction}

Cultural heritage $(\mathrm{CH})$ restoration is an extremely complex activity since it involves the expertise of several professional figures (restorers, architects/engineers, art historians, chemists, 
photographers), who produce a massive amount of documentation. All of the temporal steps of the restoration process (analysis, study, intervention) need to be documented in detail. The accessibility of these data is crucial both for a specific restoration as well as for future actions. The same data is crucial for the maintenance activities.

Many different approaches have been proposed and used to encode, store and manage restoration documentation (see Section 3 ). Most of them are based on two-dimensional (2D) approaches where the information is mapped on several 2D projections (images or drawings) and the documentation is indexed or represented by the means of those representations. However, the use of a digi-tal 3D representation would introduce significant improvements, as it would allow for the inclusion of a comprehensive and holis-tic perspective, which is inherent to any architecture and sculpted monument. The shift from the $2 \mathrm{D}$ to the 3D based model of interac-tion would overcome the limits of merely text-based descriptions of the already available information and documentation of the mon-ument, which usually provide indirect or unclear links to the other documentation.

Due to the reduction of 3D digitization costs and the progressive democratization of related technologies, the production of a 3D model is nowadays becoming a standard action in many restoration projects. Nevertheless, the potential of 3D models has not yet been completely exploited in restoration as the basis for the implementation of a reliable data documentation pipeline and as an interface to easily access them [1]. The restoration of Neptune's Fountain in Bologna (Italy) has represented the occasion to conduct a concrete case study to implement this hypothesis.

The paper discusses the result of a process of the design and implementation of a web-based IS, centered on a high-quality, 3D digital representation of the artwork that becomes the pivotal element of interaction to store and consult new and previous restoration data.

This model of interaction introduces a new approach to the design of the ISs for the consultation and production of information in restoration where data can be stored and annotated on an intuitive 3D web-based interface accessible to the entire restoration staff.

The IS presents many innovative features:

- it is built on top of a semantically-organized and high-resolution digital 3D representation of the monument to be restored;

- all system functionalities are showed to the users using standard web technologies so that the system is accessed and edited using only a browser on desktops, laptops or tablets devices;

- users can easily navigate the digital 3D model;

- all documents and data are inserted in the system with a simple interactive Graphic User Interface (GUI) and associated to the 3D model;

- the 3D model may also be used to support the online production of technical drawings depicting the conservation status and the relative intervention.

A database is used to store and access the structured and 3D annotated data and to solve user queries. The system supports all phases of the restoration process (preliminary visual analysis; uploading documents and results of scientific analysis; production of observation and intervention mappings; and data retrieval).

The paper describes the system specifications and its features by referring to the specific case study of Neptune's Fountain. However, most of its components might be easily reused to support the restoration of diverse art typologies. The early validation conducted with experts and restorers of the ISCR largely proved the effectiveness of the proposed approach.

\section{State of the art}

Over the last decades, the application of digital technologies to the production and management of $\mathrm{CH}$-related data has progressively been replacing traditional methods of data gathering, organization and management of information.

One of the main directions of work has been the exploitation of the same principle used by Geographical information systems (GIS), where layers of data are spatially associated to a territorial area. This GIS approach has been used to characterize the surface of the artwork, by linking available knowledge (e.g. all the results of the scientific investigative analysis) to specific areas defined over the artwork surface.

An example of a system following this approach is SICaR Web $[2,3]$, a web-based system for the management of the information gathered during restoration planning and the intervention phases (Fig. 1a). The data available are mapped and referenced on a 2D representation of the artwork. SICaR allows for the linking of all the data gathered during the phases of the analysis and the restoration process, e.g.: raster images, documents in TXT format, hypertext (HTML) or semi-structured text (XML).

As in any other GIS platform, SICaR offers features to crosscompare different layers (overlay function) and to support the analysis of the data.

The GIS approach (data mapped over 2D projection spaces) might be perceived as a limitation for some $\mathrm{CH}$ applications. GIS technology was originally developed to represent "2D 1/2 models" (i.e. terrain models) and is a natural choice to represent $2 \mathrm{D}$ domains (e.g. painted surfaces, or facades of buildings which can be easily projected on a plane).

More recently, GIS systems have been extended by supporting also the direct representation of 3D domains. For an example, please see the work done by Dell'Unto et al. [4] in the field of archaeology. This work extends the capability of the system and its flexibility of use from using projection planes to drawing regions directly over the 3D surface of the artwork. A more systematic approach to the use of 3D GIS was proposed by Campanaro et al. [5] through the creation and management of 3D GIS thematic layers to record and analyze specific aspects related to the state of conservation. However, its application is essentially limited to architectural spaces, where the surfaces of interest are essentially nearly planar. Additionally, built heritage is a field where conservation and management methods are more standardized [6]. Further experiments on archeological data are reported in De Reu et al. [7] and are referred to the creation of GIS-like annotations.

Moreover, the complexity of use and the time required to produce the documentation are the most critical and limiting factors of the GIS-based approach. GIS systems are usually very broad and complex in terms of functionalities as they are designed to support a wide range of different application domains. A restorer could use a very small fraction of the available features at the cost of a quite steep learning curve. Moreover, while web GIS systems are becoming more popular, they are still quite limited in terms of features, and hard to handle.

In a few cases, restorers have directly used a 3D modeling system (e.g. AutoCAD) to import 3D models and characterize the surface of the represented model. Again, this approach requires the restorer to be able to master a (complex) CAD modeling tool, whose features are not specialized. Moreover, the management of the very complex and high-resolution models available in $\mathrm{CH}$ can be complicated. An example can be found in the work done at ISCR when managing the drafting related to the very recent restoration of the Coliseum in Rome (see Fig. 1b).

Other systems also use a web platform to produce drawings or characterizations over a 2D raster image. An example is Modus Operandi, a documentation system developed by Culturanuova s.r.l. 


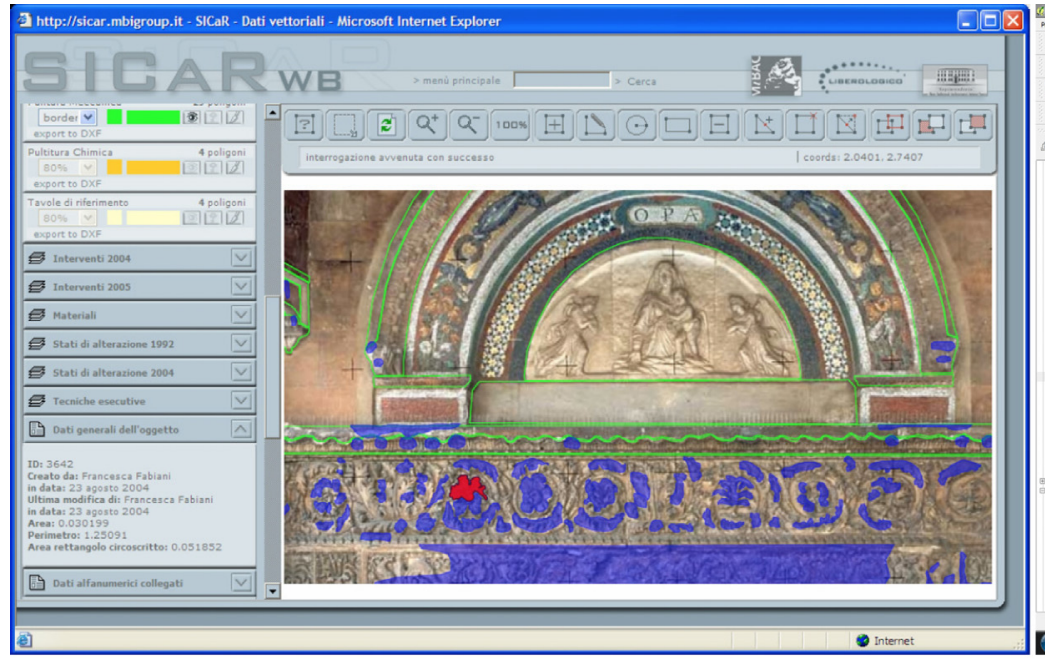

(a)

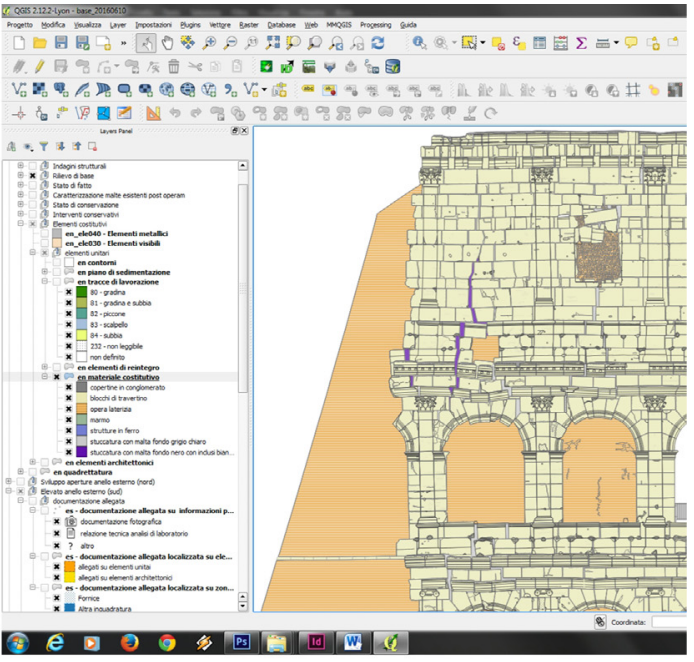

(b)

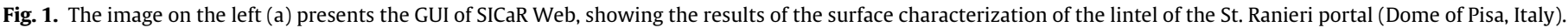
The image on the right (b) presents the results of a single surface characterization performed using AutoCAD for the Coliseum restoration (Rome, Italy).

in collaboration with a primary restoration institution, Opificio delle Pietre Dure - OPD [8]. The system can easily produce a structured report of all the data gathered in a restoration project; it also supports publishing and consultation of the data on the web.

As we will clarify in Section 5, our approach is to structure a restoration documentation system based on full 3D representations, and not on 2D views. Geometric annotations of 3D models have already been explored in research through the application of several solutions involving different ontology schemes [9] and through the conduction of some experiments [10] on the way in which 3D surfaces may be annotated. A practical implementation is the system proposed by $\mathrm{Yu}$ and Hunter [11]. The system has a similar structure to the one proposed by this paper, with the following two main differences: it is not a web-based tool and the annotation is made on a triangulated single-resolution model.

A more recent attempt along this direction is the system developed at the University of Granada [12]. It adopts a discrete 3D representation approach: the digital model used is a raster model (using voxels instead of triangles) and all surface characterizations are performed by assigning values to the surface voxels. Using a discrete voxel-based representation has some positive advantages (implementing annotation and areas selection is easier) but also some disadvantages (larger memory use).

A different approach is proposed in the NUBES project developed by CNRS-MAP [13]. In this approach, the 3D model is generated from 2D images: annotations and locations are marked on any image and automatically propagated to all other images and 3D data. A disadvantage of this approach is that drawing on 2D may generate some ambiguity in all those cases where the depicted surface has a complex shape. A web-based extension of this approach has been proposed by Stefani et al. [14], but the annotation modality still relies on unfolding the textures of the 3D model. Hence, the usability in the case of non-architectural models is low and the interaction for the end users unclear.

\section{The restoration of the Neptune complex}

Neptune's Fountain (Fig. 2) is one of the main monuments in Bologna and probably the most iconic one. Built for public use between 1563 and 1567, the fountain celebrated the papal munificence. The water flows from 38 nozzles inserted in bronze sculptures. The design of the fountain is symmetrical, with groups of figures (sirens riding dolphins, lion heads, engraved plaques, scallop shells and monster masks, shells coming out of sorrowful masks, garlands terminating in winged capriform heads, coats of arms, putti and winds) gradually leading to the figure of Neptune [15]. The whole structure is impressive in terms of size (the statue of Neptune alone is roughly $4.3 \mathrm{~m}$, while the wet area is approximately 250 square meters), quality of details, engineering and hydraulic solutions.

Nowadays, the lack of proper maintenance has strongly degraded the state of the Fountain. For this reason, the Municipality of Bologna decided, in 2016, to fund a restoration campaign focused on all the components of the monument (the sculpted elements, the hydraulic system and the masonry and stone structure). A long-term monitoring campaign is part of the future actions as well.

With this aim in mind, the University of Bologna, ISCR and ISTICNR have been engaged to design and develop a comprehensive IS that should be used on-site to collect all the documentation and data acquired and produced during the restoration process by a heterogeneous team of more than 40 experts.

Starting from the input provided by the ISCR team and by the other experts involved in the preliminary investigations, the ISTICNR and the University of Bologna designed the 3D-centered, webbased IS that was used during the last restoration project of the Fountain.

\section{Advantages and challenges of a 3D-centered, web-based system}

A restoration campaign is a complex operation that involves a number of professional figures (i.e. restorers, art historians, photographers, architects/engineers, chemists). Those professionals (indicated from now on with the term restorers) produce a large amount of data that can be defined as ". . .the systematic collection, creation, structuring and ensuring of access to information from investigations, interventions, monitoring, and maintenance. Docu-mentation formats include text, image, graphic and an increasing variety of data formats generated by examination, analytical and recording techniques" ([16], p. 280). By itself, documentation plays a central role in the effective management of the artwork; consequently, data should be handled in a unified way by the users to promote an integral first step in the planning, analysis, 


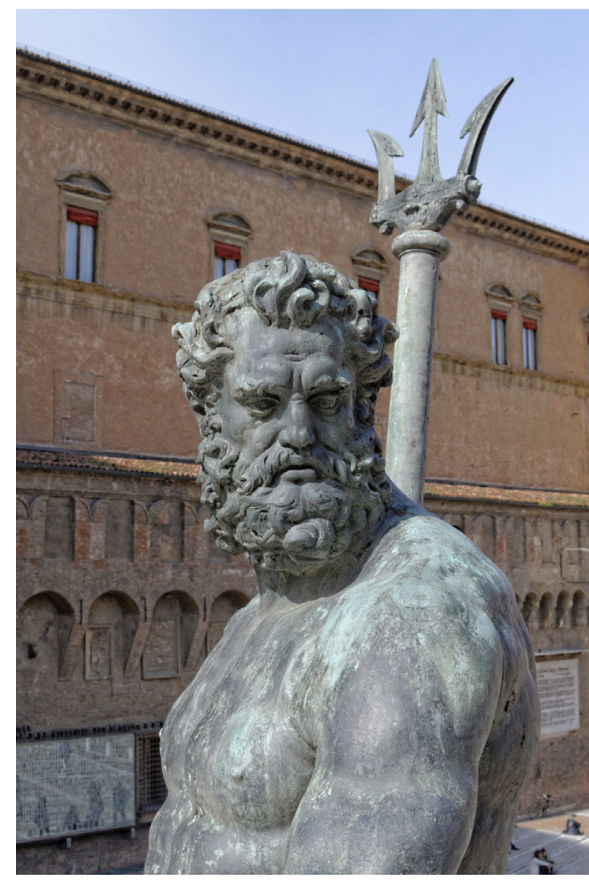

(a)

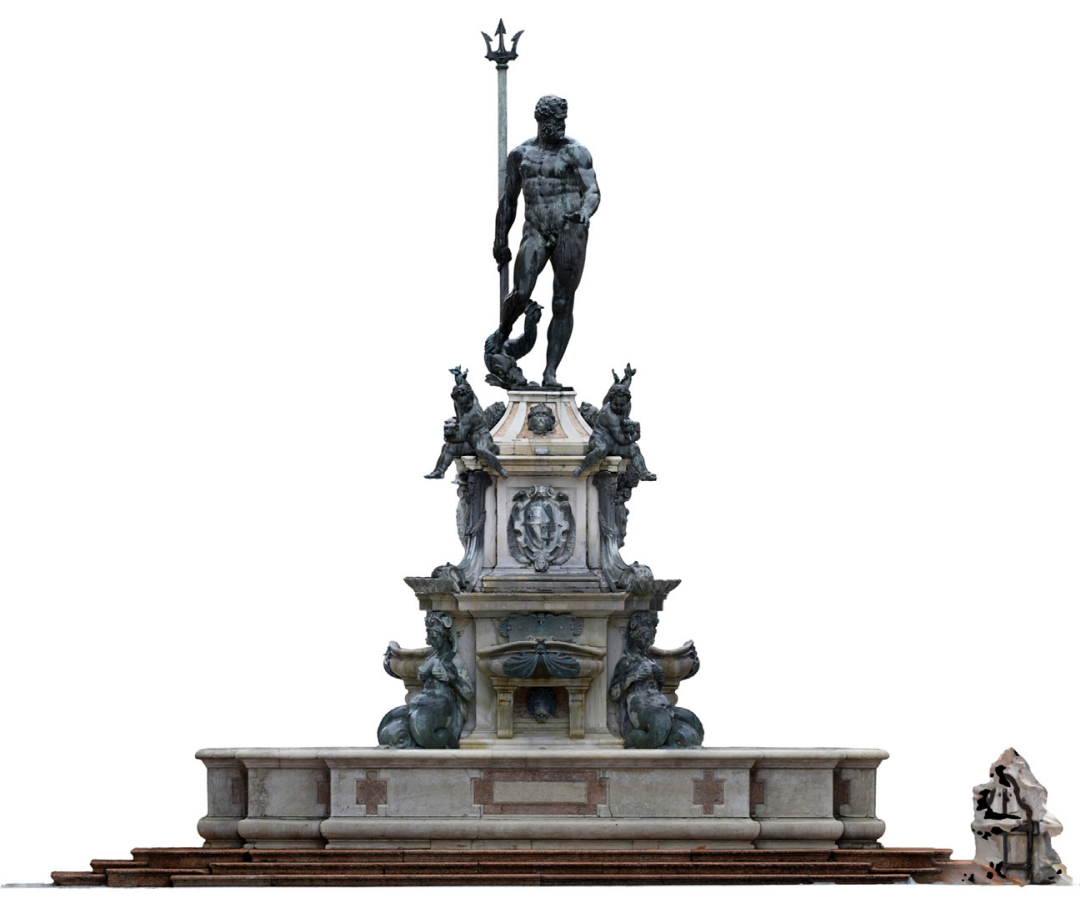

(b)

Fig. 2. Two images of the Neptune Fountain (Bologna, Italy).

intervention, and monitoring phases of conservation work. Historians and conservation scientists document most of their study and analysis by recording them in relation with the object. In addition, conservators and restorers need to represent their visual examinations and activities usually using thematic maps on the surface of the object itself.

Adopting a web-based IS, centered on 3D Models, has several advantages; among them:

- providing access to several users from different locations at the same time (even from the scaffolding);

- offering a central storage and access facility for the whole documentation;

- avoiding the need of installing any local application: the system should be accessible using a standard browser, with 3D content managed with the WebGL graphic libraries;

- mapping the information with great accuracy on 3D models;

- allowing for complex queries to retrieve all of the information easily.

Given the clear advantages described above, several challenges arise from both the research and the implementation points of view. The system should not only fulfill all of the restorers' needs, but also be flexible enough to be used in different restoration contexts. Hence, it should:

- be structured to store and present all types of restoration-related data;

- ensure a high level of usability (short training sessions, follow consolidated practice);

- require high-quality 3D models (geometry and color) since users will need access to very small details;

- provide high quality visual feedback even when the available bandwidth is limited (multi-resolution rendering);

- enable powerful and flexible search and retrieval and exploration features to be able to easily access and extract data from the final documentation.

\section{The architecture of the information system}

The challenges outlined in the previous section guided the development of the IS. The system has been designed using HTML5, thus providing the resources for an effective web-based GUI and an efficient visualization of 3D models.

\subsection{The semantic structure}

Neptune's Fountain, with 43 bronze elements, over 200 marble elements, a masonry structure underneath and a complex hydraulic system requires some semantic structuring of the 3D model to be properly managed. This is not only to represent the differences in terms of materials and functions, but also to allow for an efficient visualization and to structure the information appropriately and accurately.

Hence, the fountain has been analyzed to identify the characteristic features of its logical and structural elements. The technique used to accomplish this task could be easily extended to any building or monument typologically different from a fountain, but characterized by a complex shape or composition.

The elements have been organized in a hierarchical structure following their nature and their spatial arrangement in layers, and each individual element has been labeled using a shared lexicon and avoiding cases of ambiguity.

Fig. 3 shows the hierarchical segmentation of Neptune's Fountain. Starting from the square pavement, defined as base elevation, the fountain has been divided according to different height levels: the hypogeum (S1, i.e. the underground galleries leading to the interior of the Castellum) and the six levels (LO-L5) of the Castellum above the ground level. Within each single element of the first hierarchical order (S1-L0-...-L5), the elements of the second order have been identified. These elements have been labeled using their orientation towards cardinal directions, distinguishing between stone and metal elements. In the following, the first hierarchical order is reported: 


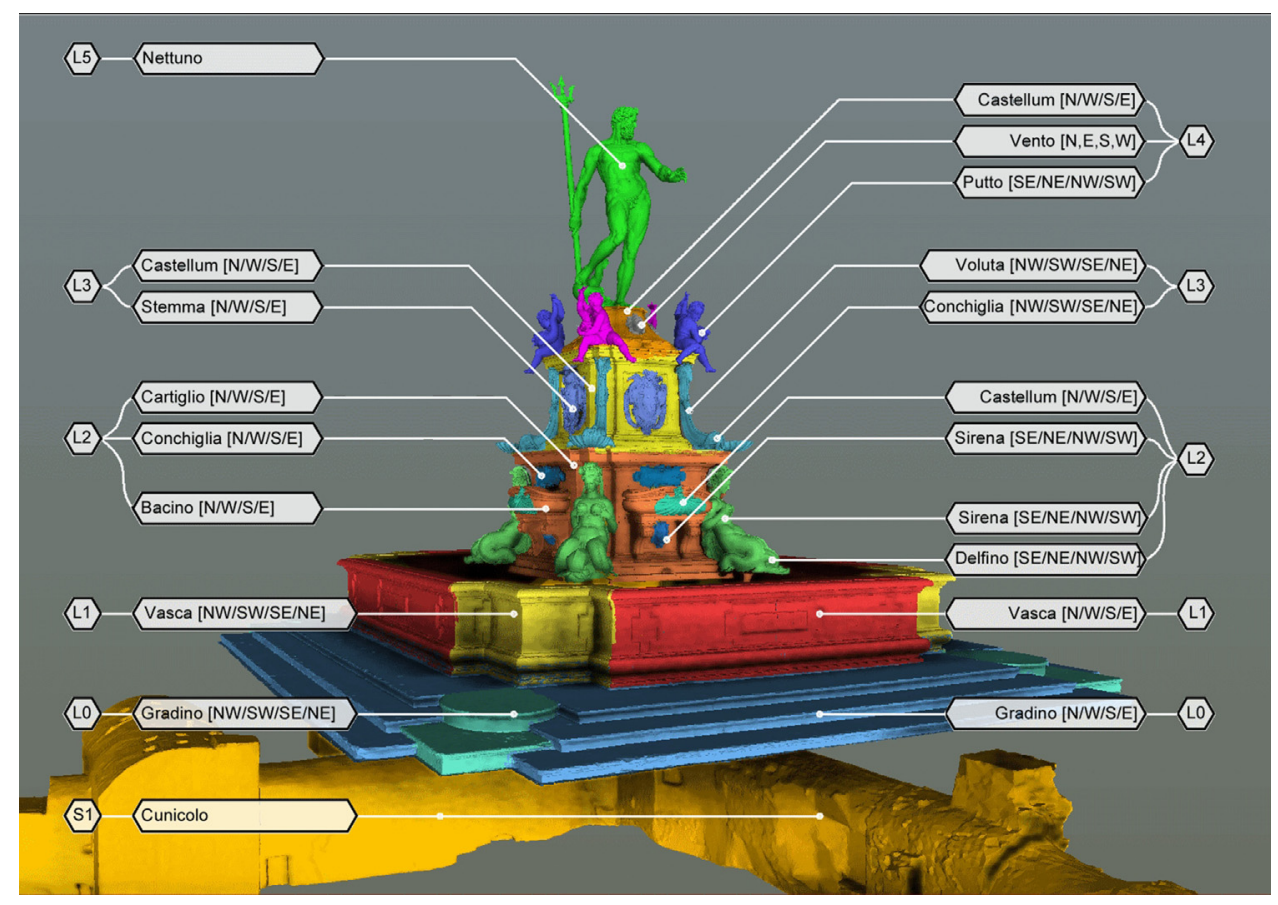

Fig. 3. Hierarchical segmentation in levels of Neptune's Fountain and the subsequent subdivision in sub-components over each level.

- S1 layer hosts the power and water systems, and the subterranean gallery;

- L0 layer corresponds to the three steps, split in sides and angle elements according to their alignment to cardinal directions, plus the small fountain visible on the side of the structure.

- L1 layer is composed by the large tub (again, divided in sides and angle elements) at the base of Castellum;

- L2 layer, the lower Castellum, is composed by the four sides of the stone structure bearing four sirens riding on dolphins, four lion heads and four plaques with inscriptions sprouting water into four stone basins decorated with scallop shells and monster masks;

- L3 layer is composed by the four sides of the stone structure of the middle Castellum, which bears four coats of arms, four garlands terminating in winged capriform heads and the set of four large bronze shells coming out of sorrowful masks;

- L4 layer contains the four sides of the stone Castellum top, two pairs of female and male Putti with dolphins in their arms and the four heads of the Winds, aligned to cardinal directions;

- L5 layer, on top of Castellum, is the sculpture of Neptune, which includes a dolphin, located underneath his right foot and the contested rod with the trident held in his right hand.

This semantic structure, based on how restorers organize their work and documentation, has guided most of the implementation of the system, as the next sections will show.

A video of the exploration of the semantic structure is accessible at this link: https://youtu.be/xTZZUQ95VQI.

\subsection{The $3 D$ digitization}

The central resource of the documentation system is a highresolution 3D model, which should encode both geometry and color information at a high level of accuracy and density. Restorers specified that a resolution of $1 \mathrm{~mm}$, both in terms of geometry and color information, would be needed for an accurate annotation. Hence, while the size of the object would call for the use of a terrestrial laser scanner (TLS) (ToF or phase-shift), we needed 3D digitization technologies to provide a denser and more accurate sampling.

While, in theory, it is possible to have a $1 \mathrm{~mm}$ resolution with a TLS, the data is generally noisy, and it would have been impossible to cover most of the hard-to-reach and higher areas of the fountain. Differently from other cases where TLS data have been used to fill missing portions of the model [17], in our case the TLS model has been used only in reference to finding an initial alignment between the different acquired portions. This ensured a coherent accuracy of data throughout the model.

Overall, a multi-scale acquisition strategy has been used: each single component has been acquired with an Eva Artec Scanner by University of Bologna and SME MCM studio; few portions of the monument have been acquired using the multi-view stereo approach (the three steps, see LO in Fig. 3); and a TLS acquisition has been used as a reference to strengthen the global alignment. The Eva Artec device provides an average resolution of less than $1 \mathrm{~mm}$ and, thanks to its hand-held capabilities, it could reach difficult/hidden areas.

The 'global' model of the fountain, obtained by aligning all the different elements, is around 610 million triangles. Additionally, the system also uses separate models for all of the different hierarchical levels of the fountain and its components (see later). For each of these element models, a 'context' model, which represents the area surrounding each element and structure, is also available. The total number of 3D models managed by the system is around 160 , for a total triangle count of more than one billion.

Since the color information acquired by the 3D scanners was below the required quality threshold, a professional photographer has provided a photographic coverage for each element. The images have been then aligned on the 3D model [18] and mapped on its surface by producing high-resolution textures $(8 \mathrm{k} \times 8 \mathrm{k})$ [19]. The quality of geometry and color has been considered sufficient by the restorers for the annotation work.

The entire 3D data processing has been done using MeshLab, an open-source software for the visualization and editing of highresolution 3D models developed by CNR-ISTI [20]. 


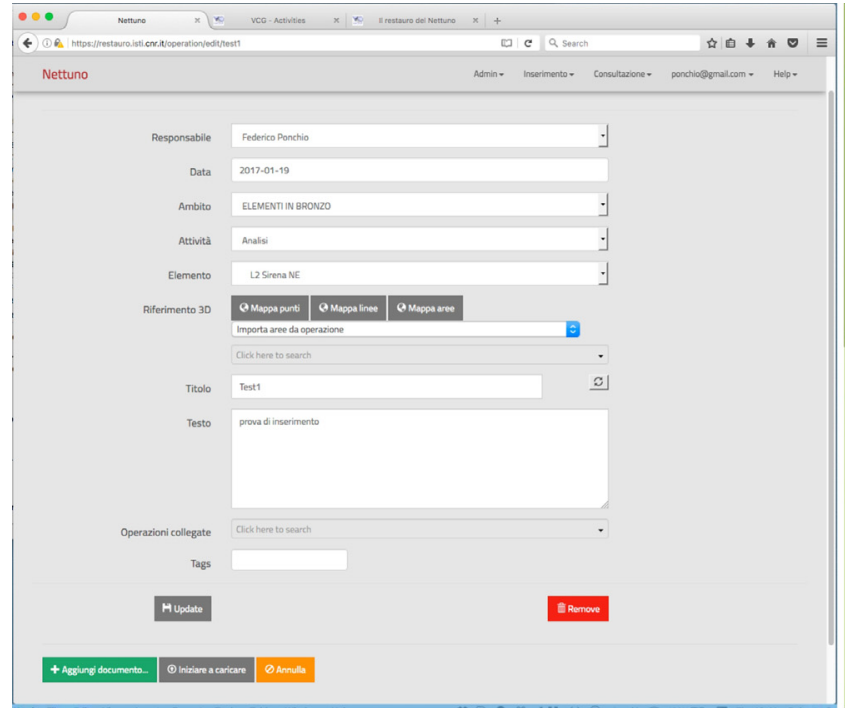

(a)

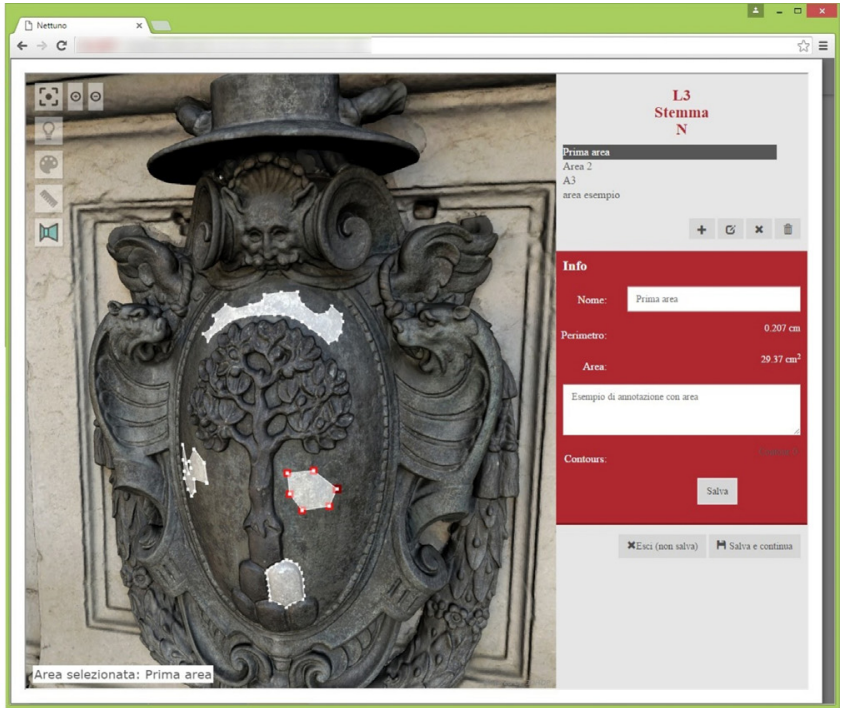

(b)

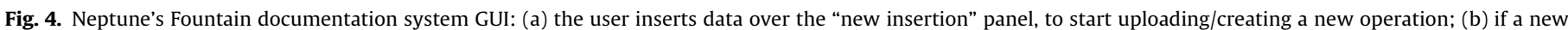
mapping has to be added, the corresponding editing window is activated.

\subsection{The Information conceptual model}

The system had to be conceptualized to model the workflow of the restorers and cover all the phases and data types involved [21]. The final structure of the workflow model rotates around the concepts of operator and operation: the operator is the data provider (or consumer, when a query is made), while the operation (Fig. 4a) is the single action aimed to record data or information. The concept of operation accounts not only for the work of restorers, but also for the contribution of any other professional (e.g., a photographer can store all the images of an acquisition campaign, or the art historian can upload the historical documentation about previous restoration interventions).

An operation requires to digit all the data needed for basic indexing, like dates, work phases, people involved, and location on the fountain complex, making it a general, multi-purpose container, suitable for all the possible annotations/work. This choice provides a uniform access to the annotations, while each operation can then contain specialized data.

An operation is a horizontal data paradigm: everything done on the Fountain (e.g. chemical analysis, visual assessment, cleaning acts, maintenance process) is an operation. However, it is possible to create "vertical" relationships (grouping, temporal succession), by linking operations.

Each operation will be an entry in the database. Therefore, we need to define its properties:

- the identification of the operator that created the entry; the date/time of entry;

- the selection of the framework (i.e. bronze elements, historical documentation, chemical analysis);

- the type of activity (analysis, planning, intervention, and maintenance);

- the reference to the specific element of the Fountain.

The latter implies that every operation will be associated to one of the hierarchical levels and elements of the semantic structure of the Fountain. Hence, every operation will be linked to the corresponding 3D model, enabling a spatial exploration of the documentation (see Section 6.5).
The operator can also attach files (images and pdf documents) to an operation. This allows for the inclusion of all of the photographic and historical documentation, and all the reports produced by experts. It is also possible to link an operation to other already existing operations. This can be useful to avoid duplication of data: e.g., a photographic campaign of an element, that could be useful for the analysis of several aspects, can be linked to all the operations related to those aspects. An example of the creation of a new oper-ation is available at the URL: https://youtu.be/ PTCMW5XMAYE.

While the operation-element association is already a link to the 3D model, a massive amount of information in a restoration action requires a more localized mapping on the surface of the object. This includes not only the case of thematic maps that describe the analysis or the intervention on each element, but also the indication of reference points or areas (e.g. the fountain nozzles, or the areas where samples of material were extracted). For this reason, we added a finer level to provide reference data on the 3D model, using an annotation interface (Fig. 4b). This has represented the most innovative and hardest part of the system to be implemented, as described in the next subsection.

\subsection{Navigating and annotating 3D models}

Since the 3D representation is the central item of the whole IS, visualization and interaction procedures are key elements for its overall usability. In terms of visualization, it is necessary to provide a smooth rendering even on low-cost devices. The specification, storage and visualization of the annotated information are the main tasks to be accomplished for the interactive functionalities.

\subsubsection{Visualization and navigation of 3D models}

Web-based visualization of 3D data is now possible due to the advent of the WebGL framework, finally supported by all browsers. Nevertheless, the visualization and navigation of high-resolution models is not an easy task. We decided to implement Neptune IS on the basis of 3DHOP technology [22]. 3DHOP provides a set of features essential to our needs:

- full support for multi-resolution visualization of textured models [23];

- pre-defined and configurable components for guided navigation; 


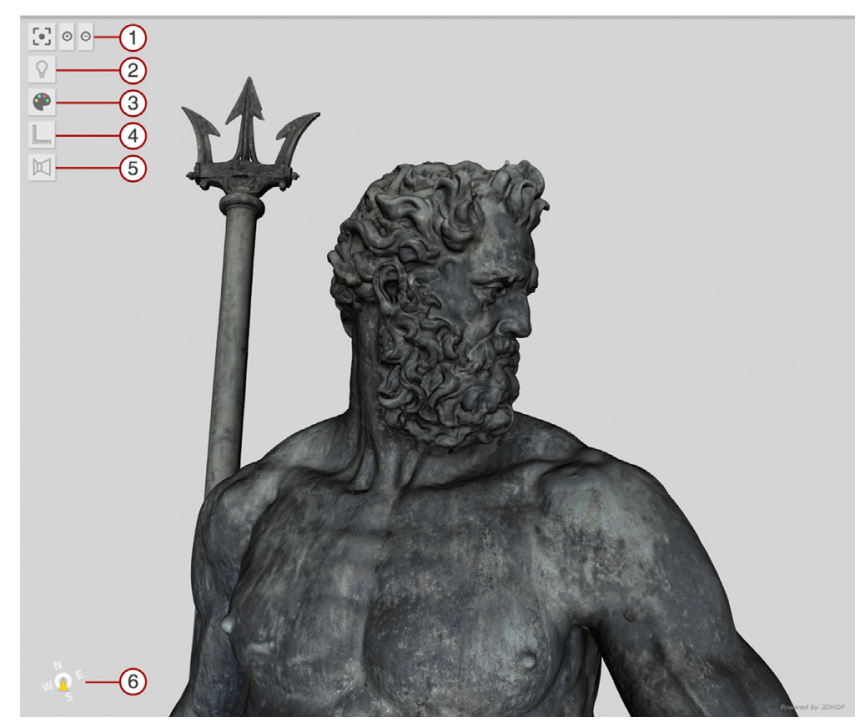

Fig. 5. The GUI of the main browsing window, with the indication of the main features.

- advanced visualization features (transparency, pre-defined views, animations);

- additional components for analysis and management (measurement, sectioning, hotspots).

Neptune IS allows for exploring the Fountain 3D model following the hierarchical organization: it is possible to work on the whole fountain, on each one of the layers, or on each element, each time using a specific 3D model.

The navigation paradigm is based on a "turntable trackball with pan" that makes it possible to rotate, zoom and pan the 3D model, while keeping the vertical axis straight.

Fig. 5 shows the 3D visualization canvas, which contains several buttons to drive the exploration (indicated with circled numbers): the reset view and zoom buttons (1); the control of the light direction (2); a button to switch on and off the texture to visualize the pure geometry (3); a button to activate an interface to take measurements (4); or to visualize the 3D model "context"(5); and finally, a compass always provides an indication of the orientation of the model (6). Fig. 6 shows the measurement and light direction functionalities.

This interface represents the best compromise between ease of use and the need to offer an extended set of interaction features, providing a seamless visualization of extremely high detailed data.

\subsubsection{Adding annotations to a $3 D$ model}

To achieve an effective 3D annotation of the restoration data, it is necessary to establish localized correspondences between the operations and the 3D surfaces.

Especially during the practical restoration activity, the so-called thematic maps represent most of the documentation, which is normally traced on a visual representation of the real object. In the standard workflow, this is often drafted over ortho-photos. These maps are produced for different types of activities, for example in the initial analysis of the conservation conditions or in the documentation of the specific intervention actions. It is crucial for restorers to be able to visualize, superimpose and compare these maps, not only to document the process, but also to have a better knowledge of the issues before starting the direct intervention. In our design, each operation can define a thematic map, establishing a strong, localized annotation.
To this aim, the system offers three different primitives for 3D annotation operations:

- points: single geometric locations in 3D space, for the documentation of localized degradation phenomena (e.g. nails, holes and small vegetation);

- polylines: lines defined over the surface, for linear structures (e.g. fracture lines or power cables);

- areas: irregular polygonal regions, used to define surface zones (e.g. circumscribing a concretion or oxidation spot).

By using these primitives, the operator may link an operation to specific location(s) of the 3D surface of the element, and create thematic maps. Each map is a set of points/polylines/areas. Most of the maps produced for the Neptune case have been produced by using area elements.

Fig. 7 shows a screenshot of the interface for inserting annotated elements (a new area element in this specific case).

The user can access this functionality directly from the interface used to create/edit a specific operation: the user indicates the type of map (points/lines/areas) and the system will automatically visualize the 3D model of the element associated to the operation in the area mapping interface. Fig. 7 shows the area mapping interface: the list of the already traced areas is presented in the upper-right part of the interface (see tag 1, Fig. 7) where the element "A8" is selected. Each area can be associated to a name and a short description (in case this is needed), while additional data (perimeter and area) are auto-matically calculated and displayed (tag 2, Fig. 7). Four small buttons (tag 3, Fig. 7) allow the user to create a new area or modify and even-tually remove it. Every single element (in this case, the areas) can be traced, even by drawing progressively several sub-portions that are then unified automatically by the system (see later). The trac-ing is intuitive, since the user simply indicates the outline of the area using a polyline, and he is guided by simple instructions in the lower-left part of the screen (tag 4, Fig. 7).

The data insertion interface has been designed to be easy to use and does not require previous experience on 3D tools. A short training, in fact, is expected to be enough for its use.

From the implementation point of view, the GUI interface for tracing points and lines is trivial. However, the tracing of areas over the 3D model was the most challenging feature. The calculation of an area indicated by a polyline on an arbitrary 3D surface is not straightforward even for a graphics tool working on a local mono-resolution data representation. In our case, we had to implement it in real time and using a peculiar 3D data representation: in each instant of time the local client has only a partial representation, at a level of detail that depends on the current view specs and hardware capabilities of the local client computer (3D data are streamed on demand, following the multi-resolution rendering library approach - therefore the $3 \mathrm{D}$ model is not really in the client memory).

The solution required a client-server approach and a nonstandard data representation. The input asked to the user is to trace the polyline and then ask to generate the area with a button. Then, a server-side service gets the set of points delimitating the region, calculates the associated area on the surface, saves it in a proper format, and sends back the data to the remote user to provide visual feedback in quasi-real-time. To compute the area in 3D space, the server calculates the intersection between the polyline and the surface of the model at the highest resolution. This intersection cannot be generated as an actual "trim" of the original 3D surface, as would be done in a local tool, because in many cases it might be a geometry which is too complex to be sent to the client (to be rendered) or to be stored in the database. Instead, the intersection is saved as a clipping volume that contains the selected surface. This volume is small enough to be effectively stored in the IS (its complexity 


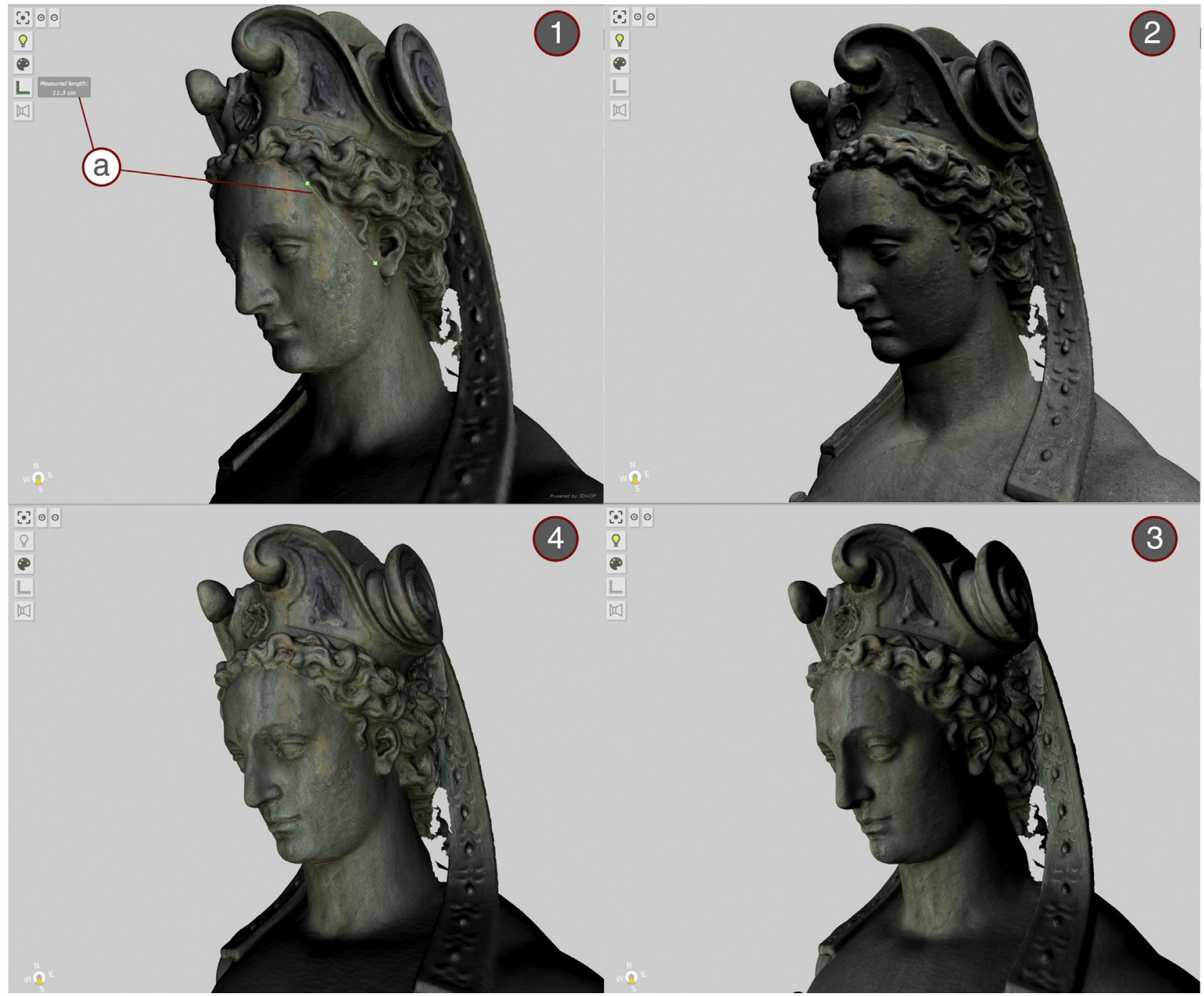

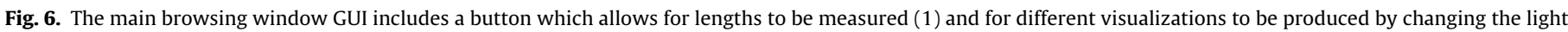
direction with the light control mode $(2,3,4)$.

is linear with the number of vertices selected in the border polyline), and can be easily sent back to the client; using a rendering technique that uses the stencil buffer, it is then possible to render the selected area with ease, even on a multi-resolution streamed model. An example of tracing of annotated data is accessible at this link: https://youtu.be/YtWG41YVxZU.

This solution gives the possibility of defining and rendering a big number of areas on a 3D model without affecting the rendering speed.

The only limitation of this approach is that the areas that have a complex shape, which cannot be entirely framed from a single point of view, cannot be directly generated. Hence, the user needs to split the area in smaller sub-portions, which are then automatically merged by the area generation component.

\subsection{Three-dimensional exploration of the information system}

The IS was designed to be something more than a pure storage facility: it was intended as a work tool and a possible reference for the long-term maintenance of the Fountain.

In order to reach this objective we needed a flexible and easy to use search and retrieval mechanism. Also in this case, in par-allel to a classic search function based on tags and queries on the database fields, we provided a 3D-based navigation of the entire documentation.

The navigation follows the hierarchical structure of the Fountain: the user can choose which layer/element of the hierarchy he wants to focus on by simply clicking on the associated volume or element tag name (see Fig. 8a).

At any time, when an element of the Fountain is shown (such as Neptune's statue, as in Fig. 8b), the list of all the operations defined on this element is displayed on the right-hand side of the navigation window. All of the operations associated to that specific element and to all its sub-elements in the hierarchy (if any) are listed, and the ones which are associated to 3D anno-tations are directly displayed on the model. Areas are currently visualized with different colors (chosen by restorers, following con-solidated documentation standards [24]), using alpha transparency to show the layering of annotations. Restorers often use other drafting modalities (i.e. hatching/screening), which might also be implemented in Neptune IS with the aid of simple shader programs. This enhanced visualization mode will be finalized once all of the data will be inserted and checked by restorers (after assessing if hatching is really needed to enhance perception of overlaid regions).

The user can switch on and off the visibility of each operation, so that the combination of any subset of the thematic maps can be 


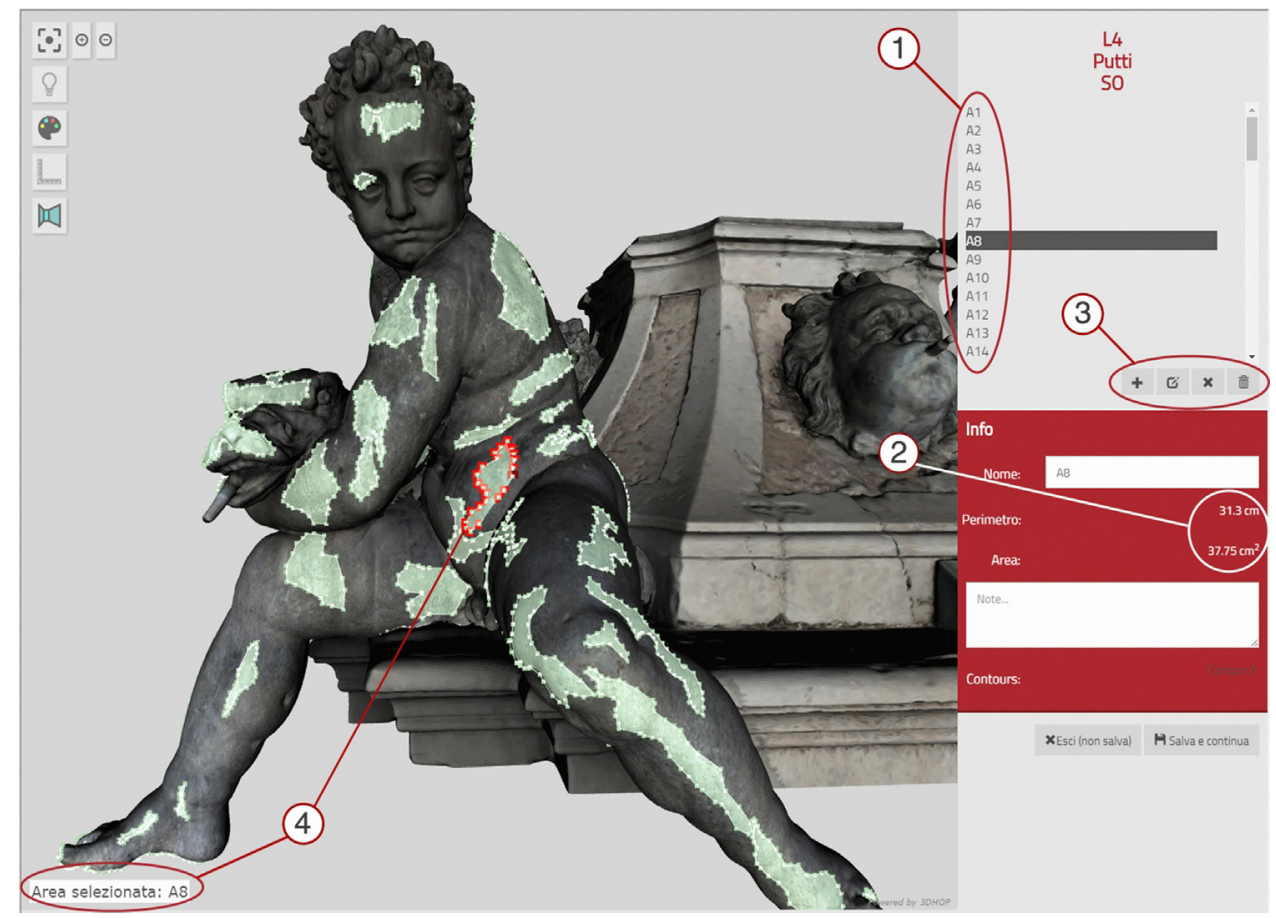

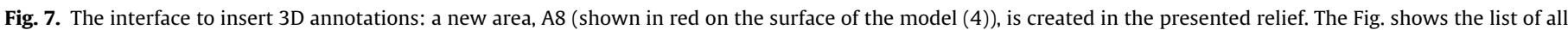
inserted areas (1), the interaction buttons (3), and the descriptive information about the area (2).

easily obtained. It is also possible to filter the list of the operations by choosing only a subset of frameworks and/or activities.

Our web-based system offers an advanced way to visualize the documentation, combining online availability, high quality data, and flexibility. An example of the exploration of the IS is accessible at this link: https://youtu.be/DwrgF9ybzhs.

The next step in the implementation of the IS will be the automatic production of technical documentation. The user will be able to choose a specific element (and thus the respective level in the hierarchical organization), a point of view, and will have to indicate which operations should be visualized on top of the respective 3D model. The system will then produce a visualization (a rendered image) and possibly will also archive of all the data indicated by the user for the production of that graphic relief.

This functionality will be finalized when the documentation will be nearly complete (see Section 7).

\section{Discussion and early assessment}

Neptune IS is the result of a complex project where different professional figures [25] collaborated to provide a tool that should not change substantially the everyday workflow of restorers, but should also provide all the advantages of new technologies, possibly adding features that were not available before.

The first activity of conceptualization and design involved a group of nearly 10 people including IT experts, restorers and architects. This led to a first prototype which was thoroughly tested by a group of three operators who provided detailed feedback for the creation of a second, more complete prototype.

The second prototype underwent a usability evaluation of 20 end users with different skills (concerning the level of proficiency in both restoration and IT themes). A preliminary short training was presented to all users, which was implemented as a single day course with frontal lessons and hands-on activities. In general, the prototype was judged complete enough to be used directly on-site.

The start-up period of use, which lasted nearly two months, was useful to find and correct some bugs and to study solutions to a few issues that emerged after real use on the restoration site. In general, the main issues were:

- the availability of a sufficiently performing internet connection: when only a low connection speed was available, the loading of the fine details of the 3D models was too slow. This problem was solved by providing the restorers with a more performing connection on site (corresponding to a standard home ADSL connection);

- operators with very basic IT skills experienced some problems in the interaction with the 3D models, and mostly in tracing annotations. To help them, a small set of video tutorials were created and made available on the platform. Those tutorials explained all the possible activities on the platform;

- the tracing of some of the thematic maps was complex due to some lack of detail in the 3D models. This was not due to the multi-resolution algorithm employed, but to the fact that some very fine details were not acquired by the scanner or the photographic campaign. This is also linked to the impressive size and complexity of the fountain: some of the elements were hard to reach or to frame with sufficient resolution. This is a common issue also when rectified photos are used, with the additional obstacle represented by the deformation introduced by the rectification of the prospective projection, which is not present on the 3D models;

- some of the elements of the fountain changed in shape or color during the restoration project, or were removed after the 3D acquisition campaign. In this case, the solution would be to acquire the shape and color at least once during the restoration campaign and again at the end. The Neptune IS structure can host different $3 \mathrm{D}$ versions of the same element, and could be quite easily extended to provide a "temporal" visualization (interactive switch among different time-tagged models). Nevertheless, performing intermediary acquisition campaigns requires a certain amount of effort and the need to stop the work of restorers, so it may not always be possible, especially in the case of projects with extremely tight schedule such as the Neptune restoration. Regardless, providing the acquisition of at least two models (one 


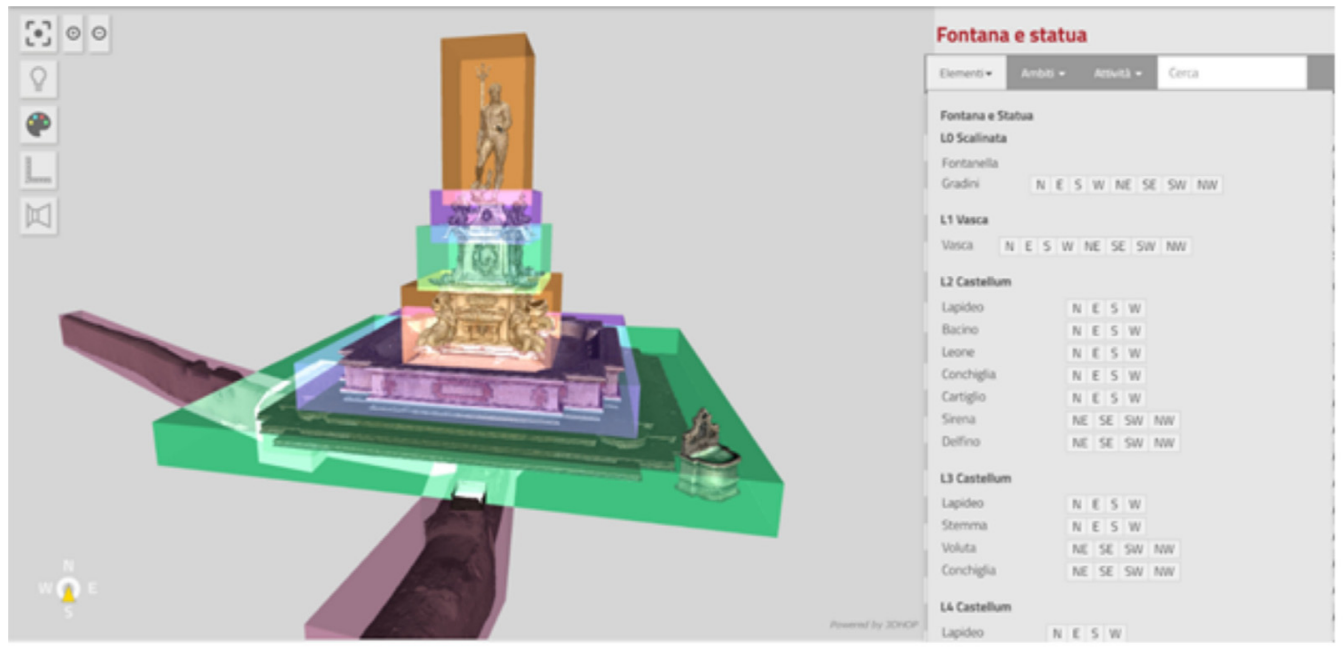

(a)

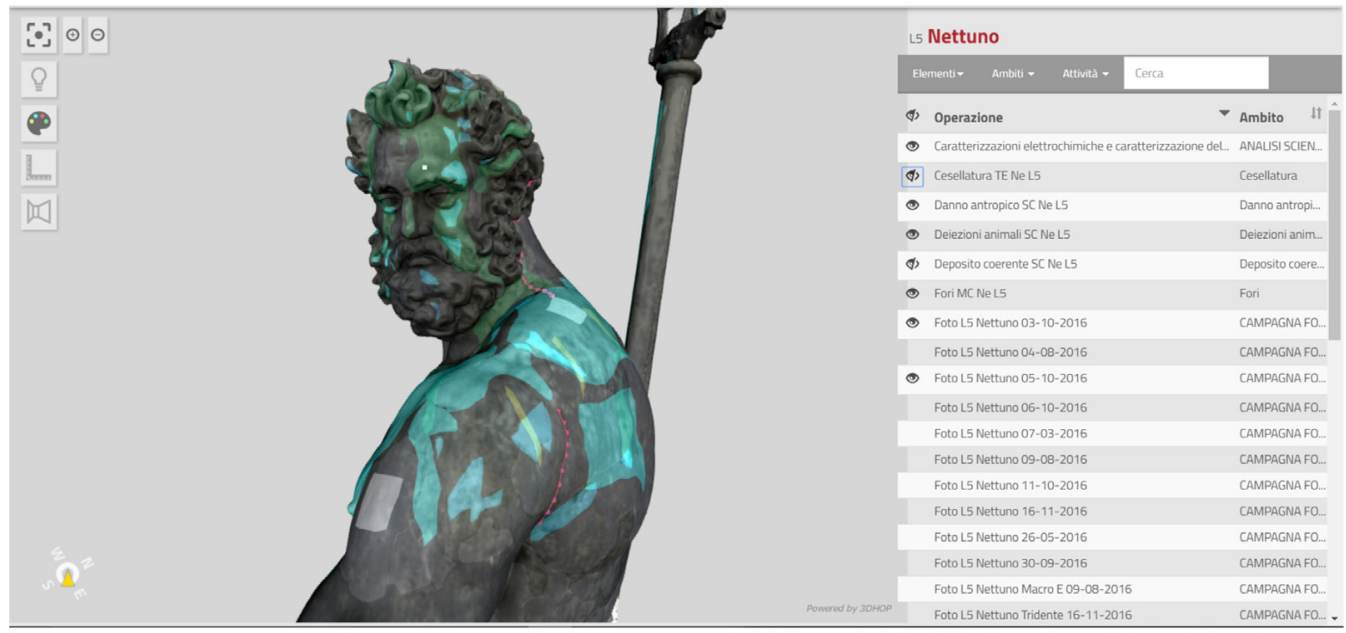

(b)

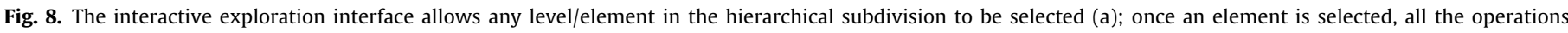
associated to a single element are accessible (b).

at the start and another at the end of the restoration) should become the standard approach. Consequently, the IS should be able to present alternative, time-tagged models. This is a feasible extension in our system. Moreover, the approach adopted for the implementation and visualization of the annotation regions (based on bounding volumes rather than on explicit geometry) makes it possible to render the same relief over multiple 3D models, each one representing a different state of the artwork;

- maintenance has been included among the types of activities associated to an operation. Maintenance operations will be used to document interventions that will take place after the end of the restoration phase. This issue is crucial to make the system usable in the long-term. Several features are necessary to this aim (multiple 3D models, linking between operations, date filtering) are already present in the project.

The system was in full-use on the restoration site in June 2016. In March 2017, a total of nearly 3000 operations were inserted, nearly 1700 of which have 3D annotated data attached and about 10,000 documents (images, reports, 2D drawings, etc.) stored. This already makes Neptune IS one of the more complex cases of restoration ISs based on 3D data.
The use of 3D was also able to provide information that further supported the work of restorers. For example, the calculation of the total surface of a thematic map allows the restorers to calculate in advance the quantity of products that will be used to clean and solve the current issues, with a strong impact in the control of costs and planning the execution time of actions.

\section{Conclusions and future work}

The paper presented the overall architecture of the IS created to support the restoration of Neptune's Fountain in Bologna and the role we have assigned to digital 3D representation in that context.

The design and implementation of the Neptune documentation system was the result of the integrated work of a team of experts belonging to different fields of work. The system has been released after only four months of intense work, due to the limited time constraint available to complete the restoration.

Regarding the 3D features, the system has been built on already consolidated elements and libraries for managing 3D data (e.g. many components belonging to the 3DHOP platform), but a massive effort has been put in place to provide access to high quality data and an easy interaction. In particular, the remote annotation 
of the 3D models called for novel uses of WebGL components and server-side mechanisms.

Although the design of the system has been strongly tailored on the peculiar and complex case of Neptune's Fountain, the main goal of this initiative has been to sketch the outline of a generic platform to be used to support any restoration project. In the case of restoration projects involving a single object, the platform already provides all the necessary tools for documenting a restoration action. In the case of complex projects, the basic platform could be a good starting point for ad-hoc implementations.

The experience with Neptune was quite successful. It opens now a new goal, the design of a cloud-based platform that should support the easy creation of a new documentation context for any new restoration project. This documentation context should offer the same functionalities presented in this paper, or in other words should be customized and offered to the users in an unattended manner (without ICT staff strongly involved in the customization of the IS). This could be one of the services of an infrastructure supporting Heritage Science and could be interconnected to other services providing a solid data preservation policy or a Virtual Research Environment.

Other directions of future work could be a better integration of photographic data (being able for example to align any new image on the 3D model, and possibly trace the thematic maps either on the 3D models or on the images), and the integration of other typologies of data (i.e. RTI imaging) and annotations.

\section{Funding}

This work was financially supported by the Municipality of Bologna.

\section{Acknowledgements}

The authors are thankful to Fabio Andreon, Manuela Faustini and Cristina Paumgardhen (Municipality of Bologna), Gisella Capponi (Director ISCR), Pier Paolo Diotallevi, Flaviano Celaschi, Gabriele Bitelli, Maurizio Mancini, Francesco Ceccarelli, Rocco Mazzeo, Giovanni Naldi, Sandra Cristino, Elisa Franzoni, Cristiana Bragalli, Claudio Galli (University of Bologna), Fabio Aramini, Dora Catalano, Lucia Conti, Giorgio D’Ercoli, Patrizia Governale, Giuseppe Guida, Giuseppina Ianiri, Francesca Romana Liserre, Anna Maria Pietrini, Sandra Ricci, Laura Rivaroli, and Giancarlo Sidoti (ISCR) for their collaboration in the project. The authors acknowledge also Giorgio Dall'Osso and Silvia Bertacchi for their contribution during the developing and testing phases.

\section{References}

[1] R. Scopigno, M. Callieri, P. Cignoni, M. Corsini, M. Dellepiane, F. Ponchio, G. Ranzuglia, 3D models for cultural heritage: beyond plain visualization, Computer 44 (7) (2011) 48-55.

[2] C. Baracchini, P. Lanari, R. Scopigno, F. Tecchia, A. Vecchi, SICaR: geographic information system for the documentation of restoration analyses and intervention, in: Proc. SPIE 5146, Optical Metrology for Arts and Multimedia, SPIE, 2003, http://dx.doi.org/10.1117/12.501505, 12 pages.

[3] LiberoLogico. com: SICaR: a web GIS for restoration projects, 2016, More info on: http://www.liberologico.com/products/.

[4] N. Dell'Unto, G. Landeschi, A.M. Leander Touati, M. Dellepiane, M. Callieri, D. Ferdani, Experiencing ancient buildings from a 3D GIS perspective: a case drawn from the Swedish Pompeii Project, J. Archaeol. Method Th. (2015) 1-22, http://dx.doi.org/10.1007/s10816-014-9226-7.

[5] D.M. Campanaro, G. Landeschi, N. Dell'Unto, A. Leander Touati, 3D GIS for cultural heritage restoration: a 'white box' workflow, J. Cult. Herit. 18 (2) (2016) 321-332.

[6] L. Toniolo, M. Boriani, G. Guidi, Built heritage: monitoring conservation management, Springer International Publishing, Switzerland, 2014.

[7] J. De Reu, G. Plets, G. Verhoeven, P. De Smedt, M. Bats, B. Cherretté, W. De Maeyer, J. Deconynck, D. Herremans, P. Laloo, M. Van Meirvenne, W. De Clercq, Towards a three-dimensional cost-effective registration of the archaeological heritage, J. Archaeol. Sci. 40 (2) (2013) 1108-1121.

[8] M. Chimenti, Modus Operandi - Documentazione informatica delle attività di conservazione e restauro (in italian), Archeomatica 2 (2010) 26-27.

[9] J. Hunter, A. Gerber, Harvesting community annotations on 3D models of museum artefacts to enhance knowledge, discovery and re-use, J. Cult. Herit. 11 (1) (2010) 81-90.

[10] S. Pena Serna, H. Schmedt, M. Ritz, A. Stork, Interactive semantic enrichment of 3D cultural heritage collections, in: VAST 2012 proceedings, Eurographics Association, 2012, pp. 33-40.

[11] C. Yu, J. Hunter, Documenting and sharing comparative analyses of 3D digital museum artifacts through semantic web annotations, ACM J. Comput. Cult. Herit. 6 (4) (2013) [Article 18, 20 pages].

[12] F. Soler, J.C. Torres, A.J. León, M.V. Luzón, Design of cultural heritage information systems based on information layers, J. Comput. Cult. Herit. 6 (4) (2013), http://dx.doi.org/10.1145/2532630.2532631, Article 15, 17 pages.

[13] A. Manuel, L. De Luca, P. Véron, A hybrid approach for the semantic annotation of spatially oriented images, Int. J. Herit. Digit. Era 3 (2) (2014) 305-320.

[14] C. Stefani, X. Brunetaud, S. Janvier-Badosa, K. Beck, L. De Luca, M. Al-Mukhtar, Developing a toolkit for mapping and displaying stone alteration on a webbased documentation platform, J. Cult. Herit. 15 (1) (2014) 1-9.

[15] R.J. Tuttle, The Neptune Fountain in Bologna. Bronze, marble and water in the making of a papal city, Brepols-Harvey Miller, New York, 2015.

[16] W. Schmid, GRADOC - Graphic Documentation Systems in Mural Painting Conservation: research seminar, Rome 16-20 November, 1999, ISBN: 92-907717-1-2.

[17] M. Callieri, A. Chica, M. Dellepiane, I. Besora, M. Corsini, J. Moyés, G. Ranzuglia, R. Scopigno, P. Brunet, Multiscale acquisition and presentation of very large artifacts: the case of Portalada, ACM J. Comput. Cult. Herit. 4 (4) (2011), Article 14,20 pages.

[18] G. Ranzuglia, M. Callieri, M. Dellepiane, P. Cignoni, R. Scopigno, MeshLab as a complete tool for the integration of photos and color with high resolution 3D geometry data, in: CAA 2012 Conference Proceedings, 2013, pp. 406-416.

[19] P. Cignoni, M. Callieri, M. Corsini, M. Dellepiane, F. Ganovelli, G. Ranzuglia, MeshLab: an open-source mesh processing tool, in: Sixth Eurographics Italian Chapter Conference, Eurographics Association, 2008, pp. 129-136.

[20] M. Corsini, M. Dellepiane, F. Ponchio, R. Scopigno, Image-to-geometry registration: a mutual information method exploiting illumination-related geometric properties, Comput. Graph. Forum 28 (7) (2009) 1755-1764.

[21] F.I. Apollonio, M. Gaiani, B. Benedetti, 3D reality-based artefact models for the management of archaeological sites using 3D GIS: a framework starting from the case study of the Pompeii Archaeological area, J. Archaeol. Sci. 39(5)(2012) 1271-1287.

[22] M. Potenziani, M. Callieri, M. Dellepiane, M. Corsini, F. Ponchio, R. Scopigno, 3DHOP: 3D heritage online presenter, Comput. Graph 52 (2015) 129-141.

[23] F. Ponchio, M. Dellepiane, Multiresolution and fast decompression for optimal web-based rendering, Graph. Mod. 88 (2016) 1-11.

[24] UNI 11182, Beni culturali, Materiali lapidei naturali ed artificiali, Descrizione della forma di alterazione: Termini e definizioni, Milano, 2006

[25] M. Matera, F. Rizzo, G. Toffetti, Web usability: principles and evaluation methods, in: E. Mendes (Ed.), Web engineering, Springer Verlag, Berlin, 2006, pp. $143-180$. 